\title{
PERISTALTIC TRANSPORT OF JOHNSON-SEGALMAN FLUID UNDER EFFECT OF A MAGNETIC FIELD
}

\author{
MOUSTAFA ELSHAHED AND MOHAMED H. HAROUN
}

Received 3 February 2005

The peristaltic transport of Johnson-Segalman fluid by means of an infinite train of sinusoidal waves traveling along the walls of a two-dimensional flexible channel is investigated. The fluid is electrically conducted by a transverse magnetic field. A perturbation solution is obtained for the case in which amplitude ratio is small. Numerical results are reported for various values of the physical parameters of interest.

\section{Introduction}

Peristalsis is an important mechanism for mixing and transporting fluids, which is generated by a progressive wave of contraction or expansion moving on the wall of the tube. The mechanism is found in the gastrointestinal, urinary, reproductive tracts, and many other glandular ducts in a living body. Considerable analysis of this mechanism has been carried out, primarily for a Newtonian fluid with a periodic train of sinusoidal peristaltic waves. The inertia-free peristaltic flow with long-wavelength analysis was given by Shapiro et al. [17]. The early developments on mathematical modeling and experimental fluid mechanics of peristaltic flow were given in a comprehensive review by Jaffrin and Shapiro [7]. The main features of the peristaltic pumping, such as trapping and reflux phenomena, have been studied extensively for Newtonian fluids. However, the rheological properties of the fluids can affect these characteristics significantly. Further, many of the physiological fluids are known to be non-Newtonian. Peristaltic transport of blood in small vessels was investigated using the viscoelastic, power-law, micropolar, Casson fluid models by $[2,14,20,22]$, respectively. Peristaltic flow of a second-order fluid in a planar channel and in an axisymmetric tube is studied by Siddiqui et al. [18, 19] under longwavelength assumption. The power-law model was used to study the fluid transport in the male reproductive tract by [24], small intestine and oesophagus by $[13,23]$.

Although there are many models to describe non-Newtonian behavior of the fluids but in recent years, the Johnson-Segalman fluid has acquired a special status, as it includes as special cases the classical Newtonian fluid and Maxwell fluid. The Johnson-Segalman model is a viscoelastic fluid model which was developed to allow for nonaffine deformations [8]. Some researchers $[9,10,11]$ used this model to explain the phenomenon 
of "spurt." "Spurt" is a phenomenon found in the flow of a number of non-Newtonian fluids in which there is a large increase in the volume throughout for a small increase in the driving pressure gradient, at a critical pressure gradient. Experimentalists usually associate "spurt" with slip at the wall and there have been a number of experiments [5] to support this hypothesis. Rao and Rajagopal [16], and Rao [15] have also been advanced towards explaining the phenomenon of "spurt." Peristaltic motion of Johnson-Segalman fluids in a planar channel was investigated by Hayat et al. [6].

The magnetohydrodynamic (MHD) flow of a fluid in a channel with elastic, rhythmically contracting walls (peristaltic flow) is of interest in connection with certain problems of the movement of conductive physiological fluids, for example, the blood and blood pump machines, and with the need for theoretical research on the operation of a peristaltic MHD compressor. The effect of moving magnetic field on blood flow was studied by Sud et al. [25], and they observed that the effect of suitable moving magnetic field accelerates the speed of blood. Srivastava and Agrawal [21] considered the blood as an electrically conducting fluid and constitutes a suspension of red cell in plasma. Also, Agrawal and Anwaruddin [1] studied the effect of magnetic field on blood flow by taking a simple mathematical model for blood through an equally branched channel with flexible walls executing peristaltic waves using long-wavelength approximation method and observed, for the flow of blood in arteries with arterial disease like arterial stenosis or arteriosclerosis, that the influence of magnetic field may be utilized as a blood pump in carrying out cardiac operations. Mekheimer [12] studied peristaltic flow of blood under effect of a magnetic field in nonuniform channels. He observed that the pressure rise for a couplestress fluid (as a blood model) is greater than that for a Newtonian fluid and is smaller for a magnetohydrodynamic fluid than for a fluid without an effect of a magnetic field.

In this paper, we study the MHD peristaltic motion of Johnson-Segalman fluid in the planar channel. A perturbation solution is obtained for the case in which amplitude ratio is small. Numerical results are reported for various values of the physical parameters of interest.

\section{Basic equations and formulation of the problem}

We will consider a two-dimensional channel of uniform width $2 d$ filled with an incompressible electrically conducting Johnson-Segalman fluid. We assume an infinite wave train traveling with velocity $c$ along the walls (see Figure 2.1). The continuity equation, the equation of motion, and the Maxwell equations governing the flow of a magnetohydrodynamic incompressible Johnson-Segalman fluid are

$$
\begin{gathered}
\operatorname{div} \mathbf{V}=0, \\
\rho \frac{d \mathbf{V}}{d t}=\operatorname{div} \Sigma+\mathbf{J} \times \mathbf{B}, \\
\operatorname{div} \mathbf{B}=0, \quad \operatorname{curl} \mathbf{B}=\mu_{m} \mathbf{J}, \quad \operatorname{curl} \mathbf{E}=-\frac{\partial \mathbf{B}}{\partial t},
\end{gathered}
$$

where $\mathbf{V}=(u(x, y, t), v(x, y, t), 0)$ is the velocity field, $\Sigma$ is the Cauchy stress tensor, $\mathbf{J}$ is 


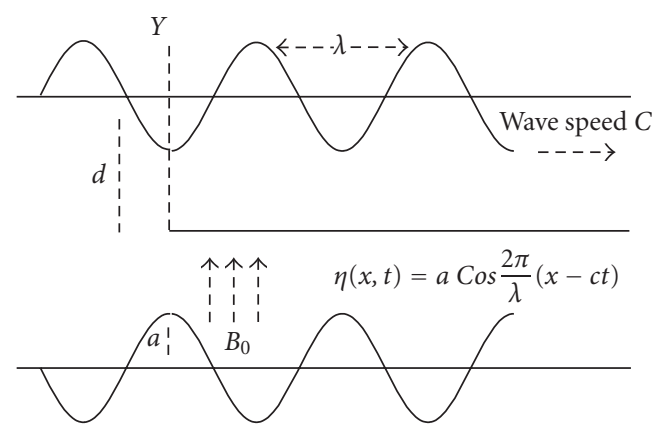

Figure 2.1. Geometry of the problem.

the current density, $\mathbf{B}$ is the total magnetic field, $\mathbf{E}$ is the total electric field, $\mu_{m}$ is the electric permeability, and $\rho$ is the density. The generalized Ohm's law is

$$
\mathbf{J}=\sigma(\mathbf{E}+\mathbf{V} \times \mathbf{B})
$$

where $\sigma$ is the electrical conductivity. It is assumed following $[3,4]$ that there is no applied or polarization voltage so that $\mathbf{E}=0$. Now we assumed that a magnetic field $\mathbf{B}=\left(0, B_{0}, 0\right)$ with a constant magnetic flux density $B_{0}$ is applied in the $y$-direction. Regardless of the induced magnetic field, it follows from (2.4) that the magnetohydrodynamic force is

$$
\mathbf{J} \times \mathbf{B}=-\sigma B_{0}^{2} u \mathbf{i}
$$

According to Johnson and Segalman [8], the constitutive equations for JohnsonSegalman fluid are

$$
\begin{gathered}
\Sigma=-P \mathbf{I}+2 \mu \mathbf{D}+\mathbf{S} \\
\mathbf{S}+m\left(\frac{d \mathbf{S}}{d t}+\mathbf{S}(\mathbf{W}-l \mathbf{D})+(\mathbf{W}-l \mathbf{D})^{T} \mathbf{S}\right)=2 \xi \mathbf{D}
\end{gathered}
$$

where, $-P I$ is the spherical part of the stress due to the constraint of incompressibility, $d / d t$ is the material time derivative, $\mu$ and $\xi$ are the viscosities, $m$ is the relaxation time, and $l$ is the slip parameter. The tensors $\mathbf{D}$ and $\mathbf{W}$ are defined as follows:

$$
\mathbf{D}=\frac{1}{2}\left(\mathbf{L}+\mathbf{L}^{T}\right), \quad \mathbf{W}=\frac{1}{2}\left(\mathbf{L}-\mathbf{L}^{T}\right), \quad \mathbf{L}=\operatorname{grad} \mathbf{V}
$$

It should be noted that this model includes the viscous Navier-Stokes fluid as a special case for $m=0$. Further, when $l=1$, the Johnson-Segalman model reduces to the Oldroyd-B fluid; and when $\mu=0$ and $l=1$, the model reduces to the Maxwell fluid. For unsteady 
two-dimensional flow, we find that (2.1)-(2.7) take the following form:

$$
\begin{gathered}
\frac{\partial u}{\partial x}+\frac{\partial v}{\partial y}=0 \\
\rho\left(\frac{\partial u}{\partial t}+u \frac{\partial u}{\partial x}+v \frac{\partial u}{\partial y}\right)=-\frac{\partial P}{\partial x}+\mu\left(\frac{\partial^{2} u}{\partial x^{2}}+\frac{\partial^{2} u}{\partial y^{2}}\right)+\frac{\partial S_{x x}}{\partial x}+\frac{\partial S_{x y}}{\partial y}-\sigma B_{0}^{2} u \\
\rho\left(\frac{\partial v}{\partial t}+u \frac{\partial v}{\partial x}+v \frac{\partial v}{\partial y}\right)=-\frac{\partial P}{\partial y}+\mu\left(\frac{\partial^{2} v}{\partial x^{2}}+\frac{\partial^{2} v}{\partial y^{2}}\right)+\frac{\partial S_{x y}}{\partial x}+\frac{\partial S_{y y}}{\partial y} \\
S_{x x}+m\left(\frac{\partial S_{x x}}{\partial t}+u \frac{\partial S_{x x}}{\partial x}+v \frac{\partial S_{x x}}{\partial y}\right)+m\left((1-l) \frac{\partial v}{\partial x}-(1+l) \frac{\partial u}{\partial y}\right) S_{x y}-2 l m \frac{\partial u}{\partial x} S_{x x}=2 \xi \frac{\partial u}{\partial x} \\
S_{x y}+m\left(\frac{\partial S_{x y}}{\partial t}+u \frac{\partial S_{x y}}{\partial x}+v \frac{\partial S_{x y}}{\partial y}\right)+\frac{m}{2}\left((1-l) \frac{\partial u}{\partial y}-(1+l) \frac{\partial v}{\partial x}\right) S_{x x} \\
+\frac{m}{2}\left((1-l) \frac{\partial v}{\partial x}-(1+l) \frac{\partial u}{\partial y}\right) S_{y y}=\xi\left(\frac{\partial v}{\partial x}+\frac{\partial u}{\partial y}\right) \\
S_{y y}+m\left(\frac{\partial S_{y y}}{\partial t}+u \frac{\partial S_{y y}}{\partial x}+v \frac{\partial S_{y y}}{\partial y}\right)+m\left((1-l) \frac{\partial u}{\partial y}-(1+l) \frac{\partial v}{\partial x}\right) S_{x y} \\
-
\end{gathered}
$$

Let the vertical displacements of the upper and lower walls be $\eta$ and $-\eta$, respectively. The geometry of the wall surface is defined as

$$
\eta=a \operatorname{Cos} \frac{2 \pi}{\lambda}(x-c t)
$$

where $a$ is the amplitude, $\lambda$ is the wavelength, and $c$ is the wave speed. The horizontal displacement will be assumed zero. Hence, the boundary conditions for the fluid are

$$
u=0, \quad v= \pm \frac{\partial \eta}{\partial t} \quad \text { at } y= \pm d \pm \eta
$$

We introduce nondimensional variables and parameters as follows:

$$
\begin{gathered}
x^{*}=\frac{x}{d}, \quad y^{*}=\frac{y}{d}, \quad u^{*}=\frac{u}{c}, \quad v^{*}=\frac{v}{c}, \quad t^{*}=\frac{c t}{d}, \quad p^{*}=\frac{p}{\rho c^{2}}, \quad \eta^{*}=\frac{\eta}{d}, \\
S_{x x}^{*}=\frac{d S_{x x}}{\mu c}, \quad S_{x y}^{*}=\frac{d S_{x y}}{\mu c}, \quad S_{y y}^{*}=\frac{d S_{y y}}{\mu c}, \quad \text { amplitude ratio } \epsilon=\frac{a}{d},
\end{gathered}
$$

wave number $\alpha=2 \pi d / \lambda$, Reynolds number $R=c d \rho / \mu$, magnetic parameter $M^{2}=d \sigma B_{0}^{2} / \rho c$, and Weissenberg number $\mathrm{We}=\mathrm{cm} / \mathrm{d}$. 
In terms of the stream function $\psi(x, y, t)$, after eliminating $P$ and dropping the asterisk over the symbols, (2.9)-(2.15) become

$$
\begin{gathered}
\frac{\partial}{\partial t} \nabla^{2} \psi+\psi_{y} \nabla^{2} \psi_{x}-\psi_{x} \nabla^{2} \psi_{y}=\frac{1}{R}\left[\nabla^{4} \psi+S_{x x, x y}+S_{x y, y y}-S_{x y, x x}-S_{y y, y x}\right]-M^{2} \psi_{y y}, \\
S_{x x}+\mathrm{We}\left[S_{x x, t}+\psi_{y} S_{x x, x}-\psi_{x} S_{x x, y}\right]-\mathrm{We}\left[(1-l) \psi_{x x}+(1+l) \psi_{y y}\right] S_{x y}-2 l \mathrm{We} \psi_{x y} S_{x x}=\frac{2 \xi}{\mu} \psi_{x y}, \\
S_{x y}+\mathrm{We}\left[S_{x y, t}+\psi_{y} S_{x y, x}-\psi_{x} S_{x y, y}\right]-\frac{\mathrm{We}}{2}\left[(1-l) \psi_{x x}+(1+l) \psi_{y y}\right] S_{y y} \\
+\frac{\mathrm{We}}{2}\left[(1+l) \psi_{x x}+(1-l) \psi_{y y}\right] S_{x x}=\frac{\xi}{\mu}\left(\psi_{y y}-\psi_{x x}\right), \\
S_{y y}+\mathrm{We}\left[S_{y y, t}+\psi_{y} S_{y y, x}-\psi_{x} S_{y y, y}\right] \\
+\mathrm{We}\left[(1+l) \psi_{x x}+(1-l) \psi_{y y}\right] S_{x y}+2 l \mathrm{We} \psi_{x y} S_{y y}=-\frac{2 \xi}{\mu} \psi_{x y}, \\
\quad \eta=\epsilon \operatorname{Cos} \alpha(x-t),
\end{gathered}
$$

where $\nabla^{2}$ denotes the Laplacian operator and subscripts indicate partial differentiation.

\section{Method of solution}

We obtain the solution for the stream function as a power series in terms of the small parameter $\epsilon$, by expanding $\psi, S_{x x}, S_{x y}, S_{y y}$, and $\partial p / \partial x$ in the following form:

$$
\begin{gathered}
\psi=\psi_{0}+\epsilon \psi_{1}+\epsilon^{2} \psi_{2}+\cdots \\
\left(\frac{\partial p}{\partial x}\right)=\left(\frac{\partial p}{\partial x}\right)_{0}+\epsilon\left(\frac{\partial p}{\partial x}\right)_{1}+\epsilon^{2}\left(\frac{\partial p}{\partial x}\right)_{2}+\cdots \\
S_{x x}=S_{x x 0}+\epsilon S_{x x 1}+\epsilon^{2} S_{x x 2}+\cdots \\
S_{x y}=S_{x y 0}+\epsilon S_{x y 1}+\epsilon^{2} S_{x y 2}+\cdots \\
S_{y y}=S_{y y 0}+\epsilon S_{y y 1}+\epsilon^{2} S_{y y 2}+\cdots
\end{gathered}
$$

The first term on the right-hand side in (3.2) corresponds to the imposed pressure gradient associated with the primary flow and the other terms correspond to the peristaltic motion. Substituting (3.1)-(3.5) into (2.17)-(2.20) and (2.22) and collecting terms of like powers of $\epsilon$, we obtain three sets of coupled differential equations with their corresponding boundary conditions in $\epsilon_{0}, \epsilon_{1}$, and $\epsilon_{2}$. The first set of differential equations in $\epsilon_{0}$, subject to the steady parallel flow and transverse symmetry assumption for a constant 
pressure gradient in the $x$-direction, yields

$$
\begin{gathered}
\psi_{0}=\frac{2 K}{R M^{2}}\left(y-\frac{\operatorname{Sinh} \Gamma y}{\Gamma \operatorname{Cosh} \Gamma}\right) \\
K=-\frac{R}{2}\left(\frac{d P}{d x}\right)_{0}, \quad \Gamma=M \sqrt{\frac{\mu R}{\mu+\xi}} .
\end{gathered}
$$

The last solution (3.6) (when $\xi$ and $M \rightarrow 0$ ) agrees with the work of Fung and Yin [26], this means that the flow at this order is independent of the viscoelastic parameter. The second and third sets of differential equations in $\psi_{1}$ and $\psi_{2}$ with their corresponding boundary conditions are satisfied by

$$
\begin{aligned}
\psi_{1}(x, y, t) & =\frac{1}{2}\left(\phi_{1}(y) e^{i \alpha(x-t)}+\phi_{1}^{*}(y) e^{-i \alpha(x-t)}\right), \\
S_{x x 1}(x, y, t) & =\frac{1}{2}\left(\phi_{2}(y) e^{i \alpha(x-t)}+\phi_{2}^{*}(y) e^{-i \alpha(x-t)}\right), \\
S_{x y 1}(x, y, t) & =\frac{1}{2}\left(\phi_{3}(y) e^{i \alpha(x-t)}+\phi_{3}^{*}(y) e^{-i \alpha(x-t)}\right), \\
S_{y y 1}(x, y, t) & =\frac{1}{2}\left(\phi_{4}(y) e^{i \alpha(x-t)}+\phi_{4}^{*}(y) e^{-i \alpha(x-t)}\right), \\
\psi_{2}(x, y, t) & =\frac{1}{2}\left(\phi_{20}(y)+\phi_{22}(y) e^{2 i \alpha(x-t)}+\phi_{22}^{*}(y) e^{-2 i \alpha(x-t)}\right), \\
S_{x x 2}(x, y, t) & =\frac{1}{2}\left(\phi_{30}(y)+\phi_{33}(y) e^{2 i \alpha(x-t)}+\phi_{33}^{*}(y) e^{-2 i \alpha(x-t)}\right), \\
S_{x y 2}(x, y, t) & =\frac{1}{2}\left(\phi_{40}(y)+\phi_{44}(y) e^{2 i \alpha(x-t)}+\phi_{44}^{*}(y) e^{-2 i \alpha(x-t)}\right), \\
S_{y y 2}(x, y, t) & =\frac{1}{2}\left(\phi_{50}(y)+\phi_{55}(y) e^{2 i \alpha(x-t)}+\phi_{55}^{*}(y) e^{-2 i \alpha(x-t)}\right),
\end{aligned}
$$

where the asterisk denotes the complex conjugate. A substitution of (3.8) into the differential equations and their corresponding boundary conditions in $\psi_{1}$ and $\psi_{2}$, we obtain three sets of coupled linear differential equations with their corresponding boundary conditions. These equations are sufficient to determine the solution up to the second order in $\epsilon$. But these equations are fourth-order ordinary differential equations with variable coefficients and the boundary conditions are not all homogeneous and the problem is not an eigenvalue problem. However, we can restrict our investigation to the case of freepumping. Physically, this means that the fluid is stationary if there is no peristaltic waves. In this case, we put $(\partial p / \partial x)_{0}=0$, which means that $K=0$, under this assumption, we get

$$
\begin{gathered}
\left(\frac{d^{2}}{d y^{2}}-\alpha^{2}\right)\left(\frac{d^{2}}{d y^{2}}-\alpha^{2}+i \alpha R\right) \phi_{1}=R M^{2} \phi_{1}^{\prime \prime}+i \alpha \phi_{4}^{\prime}-i \alpha \phi_{2}^{\prime}-\phi_{3}^{\prime \prime}-\alpha^{2} \phi_{3}, \\
\mu(1-i \alpha \mathrm{We}) \phi_{2}=2 i \alpha \xi \phi_{1}^{\prime}, \\
\mu(1-i \alpha \mathrm{We}) \phi_{3}=\xi\left(\phi_{1}^{\prime \prime}+\alpha^{2} \phi_{1}\right), \\
\mu(1-i \alpha \mathrm{We}) \phi_{4}=-2 i \alpha \xi \phi_{1}^{\prime},
\end{gathered}
$$


with

$$
\phi_{1}( \pm 1)= \pm 1, \quad \phi_{1}^{\prime}( \pm 1)=0,
$$

and

$$
\begin{gathered}
\phi_{20}^{\prime \prime \prime \prime}+\phi_{40}^{\prime \prime}=\frac{i \alpha R}{2}\left[\phi_{1}^{*} \phi_{1}^{\prime \prime}-\phi_{1} \phi_{1}^{*^{\prime \prime}}\right]^{\prime}+R M^{2} \phi_{20}^{\prime \prime}, \\
\phi_{30}=-\frac{i \alpha \mathrm{We}}{2}\left[\phi_{1}^{*} \phi_{2}-\phi_{1} \phi_{2}^{*}\right]^{\prime}+i \alpha l \mathrm{We}\left[\phi_{2}^{*} \phi_{1}^{\prime}-\phi_{2} \phi_{1}^{*^{\prime}}\right] \\
+\frac{\mathrm{We}}{2}\left[(1+l)\left(\phi_{1}^{\prime \prime} \phi_{3}^{*}+\phi_{1}^{*^{\prime \prime}} \phi_{3}\right)-\alpha^{2}(1-l)\left(\phi_{1} \phi_{3}^{*}+\phi_{1}^{*} \phi_{3}\right)\right], \\
\phi_{40}=\frac{i \alpha \mathrm{We}}{2}\left[\phi_{3}^{*} \phi_{1}-\phi_{3} \phi_{1}^{*}\right]^{\prime} \\
-\frac{\mathrm{We}}{4}\left[(1-l)\left(\phi_{1}^{\prime \prime} \phi_{2}^{*}+\phi_{1}^{*^{\prime \prime}} \phi_{2}\right)-\alpha^{2}(1-l)\left(\phi_{1} \phi_{4}^{*}+\phi_{1}^{*} \phi_{4}\right)\right. \\
\left.+(1+l)\left(\phi_{1}^{\prime \prime} \phi_{4}^{*}+\phi_{1}^{*^{\prime \prime}} \phi_{4}\right)-\alpha^{2}(1+l)\left(\phi_{1} \phi_{2}^{*}+\phi_{1}^{*} \phi_{2}\right)\right]+\frac{\xi}{\mu} \phi_{20}^{\prime \prime}, \\
\phi_{50}=\frac{i \alpha \mathrm{We}}{4}\left[\phi_{4}^{*} \phi_{1}-\phi_{4} \phi_{1}^{*}\right]^{\prime} \\
-\frac{\mathrm{We}}{2}\left[(1-l)\left(\phi_{1}^{\prime \prime} \phi_{3}^{*}+\phi_{1}^{*^{\prime \prime}} \phi_{3}\right)-\alpha^{2}(1+l)\left(\phi_{1} \phi_{3}^{*}+\phi_{1}^{*} \phi_{3}\right)\right]+i \alpha l \mathrm{We}\left[\phi_{1}^{* \prime} \phi_{4}-\phi_{1}^{\prime} \phi_{4}^{*}\right],
\end{gathered}
$$

with

$$
\phi_{20}^{\prime}( \pm 1)=\mp \frac{1}{2}\left(\phi_{1}^{\prime \prime}( \pm 1)+\phi_{1}^{*^{\prime \prime}}( \pm 1)\right),
$$

and

$$
\begin{gathered}
\left(\frac{d^{2}}{d y^{2}}-4 \alpha^{2}\right)\left(\frac{d^{2}}{d y^{2}}-4 \alpha^{2}+2 i \alpha R\right) \phi_{22}=R M^{2} \phi_{22}^{\prime \prime}+\frac{i \alpha R}{2}\left(\phi_{1}^{\prime} \phi_{1}^{\prime \prime}-\phi_{1} \phi_{1}^{\prime \prime \prime}\right)-\phi_{44}^{\prime \prime} \\
-4 \alpha^{2} \phi_{44}-2 i \alpha \phi_{33}^{\prime}+2 i \alpha \phi_{55}^{\prime}, \\
(1-2 i \alpha \mathrm{We}) \phi_{33}=\frac{4 i \alpha \xi}{\mu} \phi_{22}^{\prime}-\frac{i \alpha \mathrm{We}}{2}\left[\phi_{1}^{\prime} \phi_{2}-\phi_{1} \phi_{2}^{\prime}\right]+i \alpha l \mathrm{We} \phi_{1}^{\prime} \phi_{2} \\
+\frac{\mathrm{We}}{2}\left[(1+l) \phi_{1}^{\prime \prime} \phi_{3}-\alpha^{2}(1-l) \phi_{1} \phi_{3}\right], \\
(1-2 i \alpha \mathrm{We}) \phi_{44}=\frac{\xi}{\mu}\left(\phi_{22}^{\prime \prime}+4 \alpha^{2} \phi_{22}\right)-\frac{\mathrm{We}}{4}\left[(1-l) \phi_{1}^{\prime \prime} \phi_{2}-a^{2}(1+l) \phi_{1} \phi_{2}\right] \\
-\frac{i \alpha \mathrm{We}}{2}\left[\phi_{1}^{\prime} \phi_{3}-\phi_{1} \phi_{3}^{\prime}\right]+\frac{\mathrm{We}}{4}\left[(1+l) \phi_{1}^{\prime \prime} \phi_{4}-\alpha^{2}(1-l) \phi_{1} \phi_{4}\right], \\
(1-2 i \alpha \mathrm{We}) \phi_{55}=-\frac{4 i \alpha \xi}{\mu} \phi_{22}^{\prime}-\frac{i \alpha \mathrm{We}}{2}\left[\phi_{1}^{\prime} \phi_{4}-\phi_{1} \phi_{4}^{\prime}\right]-i \alpha l \mathrm{We} \phi_{1}^{\prime} \phi_{4} \\
+\frac{\mathrm{We}}{2}\left[a^{2}(1+l) \phi_{1} \phi_{3}-(1-l) \phi_{1}^{\prime \prime} \phi_{3}\right],
\end{gathered}
$$


with

$$
\begin{aligned}
& \phi_{22}( \pm 1)=\mp \frac{1}{4} \phi_{1}^{\prime}( \pm 1), \\
& \phi_{22}^{\prime}( \pm 1)=\mp \frac{1}{2} \phi_{1}^{\prime \prime}( \pm 1),
\end{aligned}
$$

where $\left({ }^{\prime}\right)$ denotes the derivative with respect to $y$. The solutions of (3.9)-(3.12) are

$$
\begin{aligned}
& \phi_{1}(y)=A 1 \operatorname{Sinh} \alpha_{1} y+B 1 \operatorname{Sinh} \beta_{1} y, \\
& \phi_{2}(y)=A 2 \operatorname{Cosh} \alpha_{1} y+B 2 \operatorname{Cosh} \beta_{1} y, \\
& \phi_{3}(y)=A 3 \operatorname{Sinh} \alpha_{1} y+B 3 \operatorname{Sinh} \beta_{1} y, \\
& \phi_{4}(y)=-A 2 \operatorname{Cosh} \alpha_{1} y-B 2 \operatorname{Cosh} \beta_{1} y,
\end{aligned}
$$

where

$$
\begin{gathered}
A 1=\frac{-\beta_{1} \operatorname{Cosh} \beta_{1}}{\alpha_{1} \operatorname{Cosh} \alpha_{1} \operatorname{Sinh} \beta_{1}-\beta_{1} \operatorname{Cosh} \beta_{1} \operatorname{Sinh} \alpha_{1}}, \\
B 1=\frac{\alpha_{1} \operatorname{Cosh} \alpha_{1}}{\alpha_{1} \operatorname{Cosh} \alpha_{1} \operatorname{Sinh} \beta_{1}-\beta_{1} \operatorname{Cosh} \beta_{1} \operatorname{Sinh} \alpha_{1}}, \\
A 2=\frac{2 i \alpha \alpha_{1} \xi A 1}{\mu(1-i \alpha \mathrm{We})}, \quad B 2=\frac{2 i \alpha \beta_{1} \xi B 1}{\mu(1-i \alpha \mathrm{We})}, \\
A 3=\frac{\left(\alpha^{2}+\alpha_{1}^{2}\right) \xi A 1}{\mu(1-i \alpha \mathrm{We})}, \quad B 3=\frac{\left(\alpha^{2}+\beta_{1}^{2}\right) \xi B 1}{\mu(1-i \alpha \mathrm{We})}, \\
\alpha_{1}^{2}=\frac{N+\sqrt{N^{2}-4 \alpha^{2} \beta^{2}}}{2}, \quad \beta_{1}^{2}=\frac{N-\sqrt{N^{2}-4 \alpha^{2} \beta^{2}}}{2}, \\
N=\alpha^{2}+\beta^{2}-\frac{i\left(\alpha^{2}-\beta^{2}\right) M^{2}}{\alpha}, \quad \beta^{2}=\alpha^{2}-\frac{i \alpha R \mu(1-i \alpha \mathrm{We})}{(\mu(1-i \alpha \mathrm{We})+\xi)} .
\end{gathered}
$$

Next, in the expansion of $\psi_{2}$, we need only to concern ourselves with the terms $\phi_{20}^{\prime}(y)$ as our aim is to determine the mean flow only. Thus, the differential equations (3.14)-(3.17) subject to the boundary condition (3.18) give the expression

$$
\begin{aligned}
& \phi_{20}^{\prime}(y)= F(y)+2 C 1 \frac{\operatorname{Cosh}(\Gamma y)-\operatorname{Cosh}(\Gamma)}{\Gamma^{2} \operatorname{Cosh}(\Gamma)}+(D-F(1)) \frac{\operatorname{Cosh}(\Gamma y)}{\operatorname{Cosh}(\Gamma)}, \\
& D=\phi_{20}^{\prime}( \pm 1)=-\frac{1}{2}\left[\alpha_{1}^{2} A 1 \operatorname{Sinh} \alpha_{1}+\alpha_{1}^{* 2} A 1^{*} \operatorname{Sinh} \alpha_{1}^{*}+\beta_{1}^{2} B 1 \operatorname{Sinh} \beta_{1}+\beta_{1}^{* 2} B 1^{*} \operatorname{Sinh} \beta_{1}^{*}\right] \\
& F(y)= s_{1} \operatorname{Cosh}\left(\alpha_{1}+\beta_{1}^{*}\right) y+s_{2} \operatorname{Cosh}\left(\alpha_{1}-\beta_{1}^{*}\right) y+s_{3} \operatorname{Cosh}\left(\alpha_{1}^{*}+\beta_{1}\right) y \\
&+s_{4} \operatorname{Cosh}\left(\alpha_{1}^{*}-\beta_{1}\right) y+s_{5} \operatorname{Cosh}\left(\alpha_{1}+\alpha_{1}^{*}\right) y+s_{6} \operatorname{Cosh}\left(\beta_{1}+\beta_{1}^{*}\right) y \\
&+s_{7} \operatorname{Cosh}\left(\beta_{1}-\beta_{1}^{*}\right) y+s_{8} \operatorname{Cosh}\left(\alpha_{1}-\alpha_{1}^{*}\right) y,
\end{aligned}
$$




$$
\begin{aligned}
& s_{1}=\frac{\mu\left(\alpha_{1}+\beta_{1}^{*}\right)}{4(\mu+\xi)\left(\left(\alpha_{1}+\beta_{1}^{*}\right)^{2}-\Gamma^{2}\right)} \\
& \times\left[i \alpha R\left(\alpha_{1}-\beta_{1}^{*}\right) A 1 B 1^{*}+\mathrm{We}\left(\alpha_{1}^{2}-\alpha^{2}\right) A 1 B 2^{*}-i \alpha \mathrm{We}\left(\alpha_{1}+\beta_{1}^{*}\right)\left(A 1 B 3^{*}-A 3 B 1^{*}\right)\right. \\
& \left.+\mathrm{We}\left(\beta_{1}^{* 2}-\alpha^{2}\right) A 2 B 1^{*}\right] \text {, } \\
& s_{2}=\frac{\mu\left(\alpha_{1}-\beta_{1}^{*}\right)}{4(\mu+\xi)\left(\left(\alpha_{1}-\beta_{1}^{*}\right)^{2}-\Gamma^{2}\right)} \\
& \times\left[-i \alpha R\left(\alpha_{1}+\beta_{1}^{*}\right) A 1 B 1^{*}+\mathrm{We}\left(\alpha_{1}^{2}-\alpha^{2}\right) A 1 B 2^{*}\right. \\
& \left.+i \alpha \mathrm{We}\left(\alpha_{1}-\beta_{1}^{*}\right)\left(A 1 B 3^{*}-A 3 B 1^{*}\right)+\mathrm{We}\left(\alpha^{2}-\beta_{1}^{* 2}\right) A 2 B 1^{*}\right] \text {, } \\
& s_{3}=\frac{\mu\left(\alpha_{1}^{*}+\beta_{1}\right)}{4(\mu+\xi)\left(\left(\alpha_{1}^{*}+\beta_{1}\right)^{2}-\Gamma^{2}\right)} \\
& \times\left[i \alpha R\left(\beta_{1}-\alpha_{1}^{*}\right) A 1^{*} B 1+\mathrm{We}\left(\beta_{1}^{2}-\alpha^{2}\right) B 1 A 2^{*}-i \alpha \mathrm{We}\left(\beta_{1}+\alpha_{1}^{*}\right)\left(B 1 A 3^{*}-B 3 A 1^{*}\right)\right. \\
& \left.-\mathrm{We}\left(\alpha^{2}-\alpha_{1}^{* 2}\right) B 2 A 1^{*}\right] \text {, } \\
& s_{4}=\frac{\mu\left(\alpha_{1}^{*}-\beta_{1}\right)}{4(\mu+\xi)\left(\left(\alpha_{1}^{*}-\beta_{1}\right)^{2}-\Gamma^{2}\right)} \\
& \times\left[i \alpha R\left(\beta_{1}+\alpha_{1}^{*}\right) A 1^{*} B 1-\operatorname{We}\left(\beta_{1}^{2}-\alpha^{2}\right) B 1 A 2^{*}-i \alpha \operatorname{We}\left(\beta_{1}-\alpha_{1}^{*}\right)\left(B 1 A 3^{*}-B 3 A 1^{*}\right)\right. \\
& \left.-\mathrm{We}\left(\alpha^{2}-\alpha_{1}^{* 2}\right) B 2 A 1^{*}\right] \text {, } \\
& s_{5}=\frac{\mu\left(\alpha_{1}+\alpha_{1}^{*}\right)}{4(\mu+\xi)\left(\left(\alpha_{1}+\alpha_{1}^{*}\right)^{2}-\Gamma^{2}\right)} \\
& \times\left[i \alpha R\left(\alpha_{1}-\alpha_{1}^{*}\right) A 1 A 1^{*}+\mathrm{We}\left(\alpha_{1}^{2}-\alpha^{2}\right) A 1 B 2^{*}-i \alpha \mathrm{We}\left(\alpha_{1}+\alpha_{1}^{*}\right)\left(A 1 A 3^{*}-A 3 A 1^{*}\right)\right. \\
& \left.+\mathrm{We}\left(\alpha_{1}^{* 2}-\alpha^{2}\right) A 2 A 1^{*}\right] \text {, } \\
& s_{6}=\frac{\mu\left(\beta_{1}+\beta_{1}^{*}\right)}{4(\mu+\xi)\left(\left(\beta_{1}+\beta_{1}^{*}\right)^{2}-\Gamma^{2}\right)} \\
& \times\left[i \alpha R\left(\beta_{1}-\beta_{1}^{*}\right) B 1 B 1^{*}+\mathrm{We}\left(\beta_{1}^{2}-\alpha^{2}\right) B 1 B 2^{*}-i \alpha \mathrm{We}\left(\beta_{1}+\beta_{1}^{*}\right)\left(B 1 B 3^{*}-B 3 B 1^{*}\right)\right. \\
& \left.+\mathrm{We}\left(\beta_{1}^{* 2}-\alpha^{2}\right) B 2 B 1^{*}\right] \text {, } \\
& s_{7}=\frac{\mu\left(\beta_{1}-\beta_{1}^{*}\right)}{4(\mu+\xi)\left(\left(\beta_{1}-\beta_{1}^{*}\right)^{2}-\Gamma^{2}\right)} \\
& \times\left[-i \alpha R\left(\beta_{1}+\beta_{1}^{*}\right) B 1 B 1^{*}+\mathrm{We}\left(\beta_{1}^{2}-\alpha^{2}\right) B 1 B 2^{*}\right. \\
& \left.+i \alpha \operatorname{We}\left(\beta_{1}-\beta_{1}^{*}\right)\left(B 1 B 3^{*}-B 3 B 1^{*}\right)-\mathrm{We}\left(\beta_{1}^{* 2}-\alpha^{2}\right) B 2 B 1^{*}\right] \text {, } \\
& s_{8}=\frac{\mu\left(\alpha_{1}-\alpha_{1}^{*}\right)}{4(\mu+\xi)\left(\left(\alpha_{1}-\alpha_{1}^{*}\right)^{2}-\Gamma^{2}\right)} \\
& \times\left[-i \alpha R\left(\alpha_{1}+\alpha_{1}^{*}\right) A 1 A 1^{*}+\mathrm{We}\left(\alpha_{1}^{2}-\alpha^{2}\right) A 1 A 2^{*}\right. \\
& \left.+i \alpha \mathrm{We}\left(\alpha_{1}-\alpha_{1}^{*}\right)\left(A 1 A 3^{*}-A 3 A 1^{*}\right)-\mathrm{We}\left(\alpha_{1}^{* 2}-\alpha^{2}\right) A 2 A 1^{*}\right] \text {, }
\end{aligned}
$$




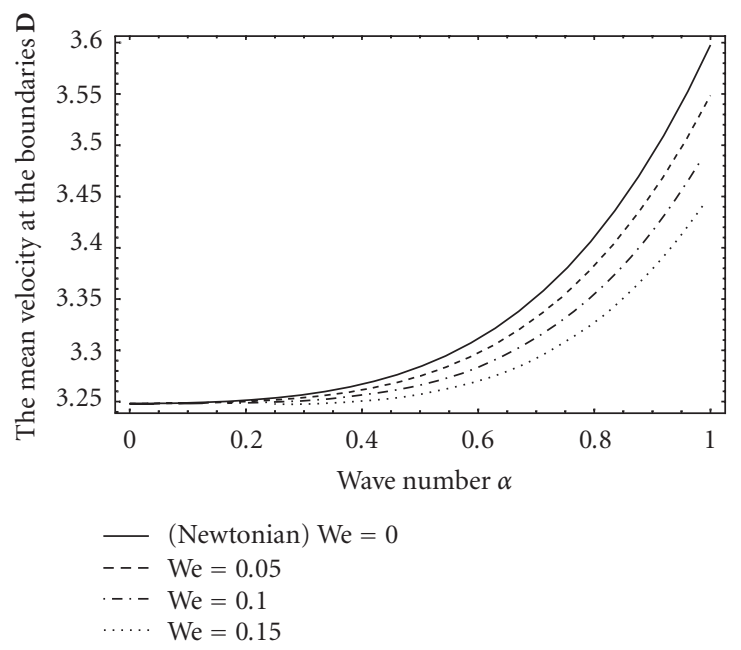

Figure 4.1. Effect of the viscoelastic parameter We on variation of $D$ with wave number $\alpha$ for $M=0.5$, $\xi / \mu=1$, and $R=10$.

Thus, we see that one constant $C 1$ remains arbitrary in the solution. Substituting (3.1)(3.5) into (2.9), and time-average equation of the second order of $\epsilon$ with assumptions that $K=0$, we find that

$$
C 1=R\left(\frac{\overline{\partial p}}{\partial x}\right)_{2} .
$$

Also, the mean time-average velocity may be written as

$$
\begin{aligned}
\bar{u}(y) & =\frac{\epsilon^{2}}{2} \phi_{20}^{\prime}(y) \\
& =\frac{\epsilon^{2}}{2}\left[F(y)+(D-F(1)) \frac{\operatorname{Cosh} \Gamma y}{\operatorname{Cosh} \Gamma}+\frac{2 R}{\Gamma^{2}}\left(\frac{\overline{\partial p}}{\partial x}\right)_{2}\left(\frac{\operatorname{Cosh} \Gamma y-\operatorname{Cosh} \Gamma}{\operatorname{Cosh} \Gamma}\right)\right] .
\end{aligned}
$$

Note that if we put the magnetic parameter $M$, Weissenberg number We, and the viscosity $\xi$ equal to zero, then the results of the problem reduce exactly to the same as that found by Fung and Yin [26] for Newtonian fluid.

\section{Numerical results and discussion}

A close look at (3.29) reveals that the mean axial velocity of a hydromagnetic flow of Johnson-Segalman fluid is controlled by viscoelastic parameter, magnetic parameter, wave number, Reynolds number, and second-order time-averaged pressure gradient. In this section, the mean velocity at the boundaries of the channel, the time-averaged mean axial-velocity distribution, and reversal flow are calculated for various values of these parameters in the free-pumping case. Numerical calculations based on (3.29) show that 


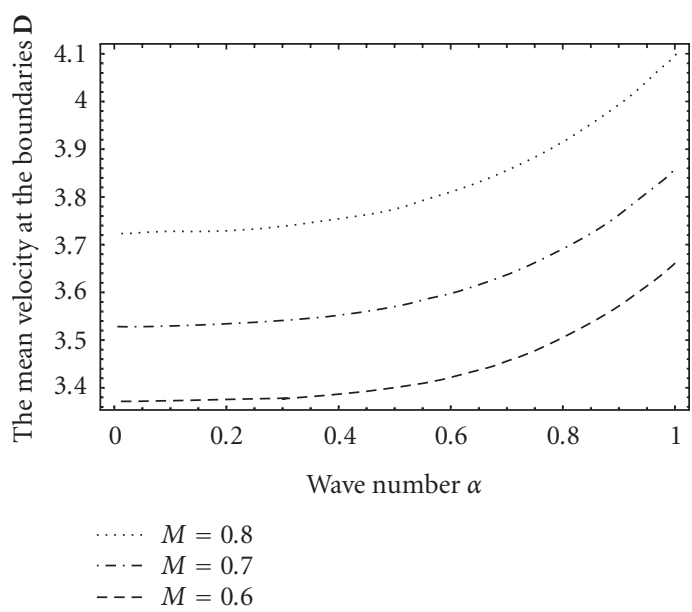

Figure 4.2. Effect of the magnetic parameter $M$ on variation of $D$ with wave number $\alpha$ for $\mathrm{We}=0.1$, $\xi / \mu=1$, and $R=10$.

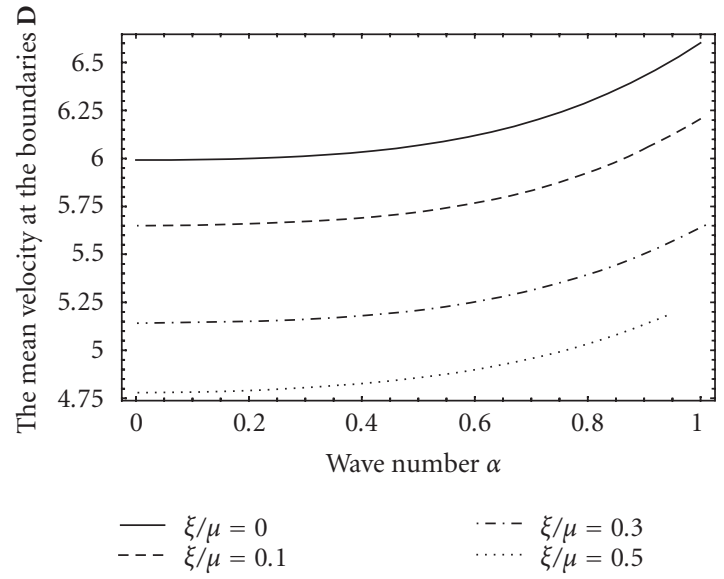

Figure 4.3. Effect of the viscosity ratio $\xi / \mu$ on variation of $D$ with wave number $\alpha$ for We $=0.1, M=1$, and $R=10$.

the mean axial velocity of the fluid due to peristaltic motion is dominated by the constant $D$ and the term $\left(2 R / \Gamma^{2}\right)(\overline{\partial p} / \partial x)_{2}((\operatorname{Cosh}(\Gamma y)-\operatorname{Cosh}(\Gamma)) / \operatorname{Cosh}(\Gamma))$. In addition to these terms, there is a perturbation term $F(y)-F(1)(\operatorname{Cosh}(\Gamma y) / \operatorname{Cosh}(\Gamma))$ which controls the direction of the peristaltic mean flow across the cross-section. The constant $D$, which initially arose from the nonslip condition of the axial velocity on the wall, is due to the value of $\phi_{20}^{\prime}(y)$ at the boundary and is related to the mean velocity at the boundaries of the channel by $\bar{u}( \pm 1)=\left(\epsilon^{2} / 2\right) \phi_{20}^{\prime}( \pm 1)=\left(\epsilon^{2} / 2\right) D$. Figures $4.1,4.2$, and 4.3 represent the variation of $D$ with $\alpha$ for various values of the magnetic parameter $M$, the viscosity ratio 


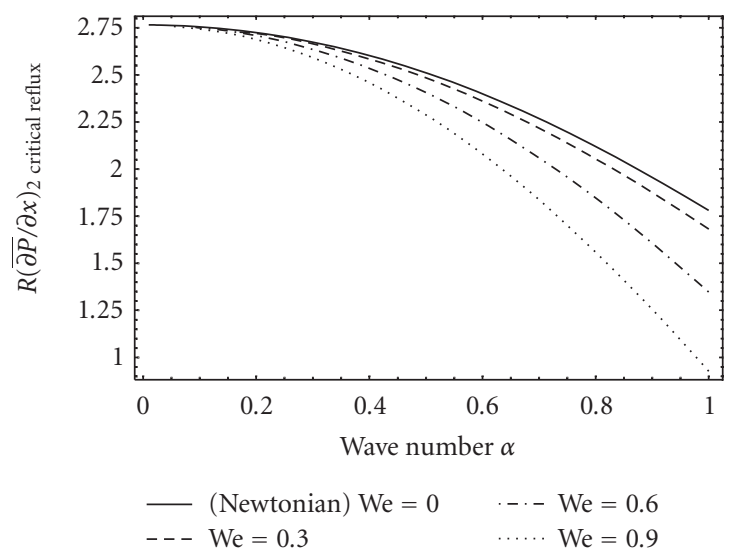

Figure 4.4. Effect of the viscoelastic parameter We on variation of critical reflux pressure gradient

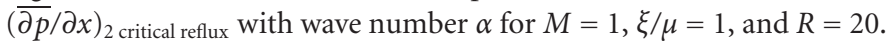

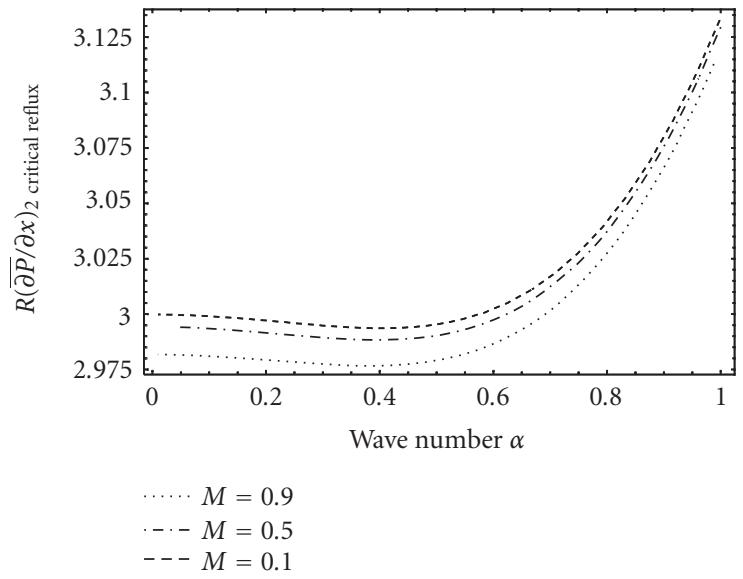

Figure 4.5. Effect of the magnetic parameter $M$ on variation of critical reflux pressure gradient

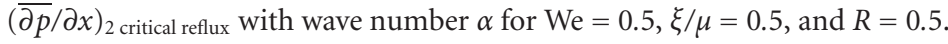

$\xi / \mu$, and the Weissenberg number We. The numerical results indicate that $D$ decreases with increasing We and $\xi / \mu$ and increases with increasing $M$ and $\alpha$. Yin and Fung [26] define a flow reflux whenever there is a negative mean velocity in the flow field. Then according to (3.29), the critical reflux condition is given by

$$
\left(\frac{\overline{\partial p}}{\partial x}\right)_{2 \text { critical reflux }}=\frac{\Gamma^{2}}{2 R(1-\operatorname{Cosh}(\Gamma))}(F(1)-F(0) \operatorname{Cosh}(\Gamma)-D),
$$




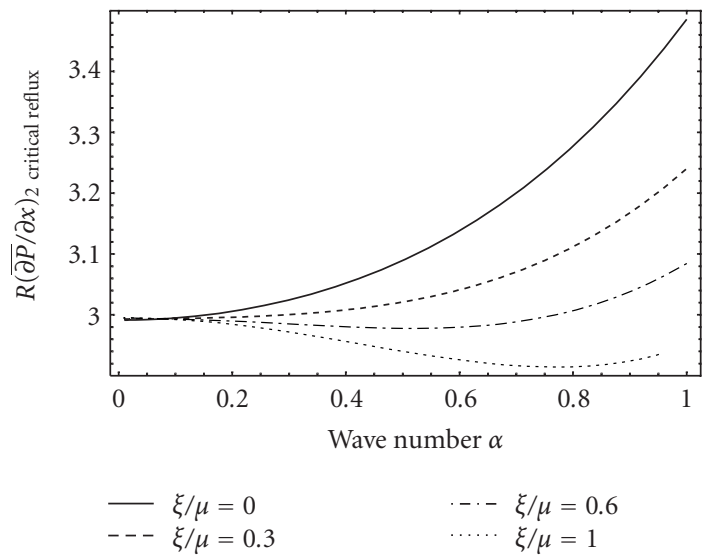

Figure 4.6. Effect of the viscosity ratio $\xi / \mu$ on variation of critical reflux pressure gradient $(\overline{\partial p} / \partial x)_{2 \text { critical reflux }}$ with wave number $\alpha$ for $\mathrm{We}=0.5, M=0.5$, and $R=0.5$.

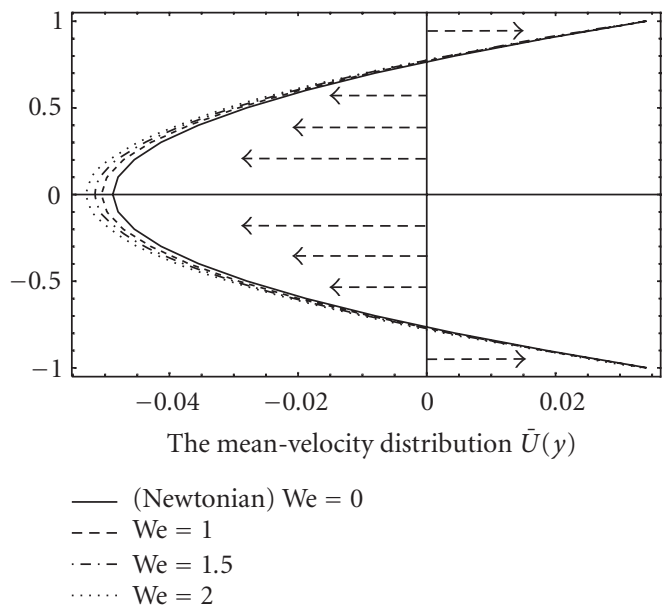

Figure 4.7. Effect of the viscoelastic parameter We on the mean-velocity distribution and reversal flow for $(\overline{\partial p} / \partial x)_{2}=1, \alpha=0.1, M=0.2, \epsilon=0.15, \xi / \mu=1$, and $R=10$.

and the reflux occurs when $(\overline{\partial p} / \partial x)_{2}>(\overline{\partial p} / \partial x)_{2}$ critical reflux. Figures 4.4, 4.5, and 4.6 represent the variation of $(\overline{\partial p} / \partial x)_{2}$ critical reflux with $\alpha$ for various values of $M, \xi / \mu$ and We. The results reveal that $(\overline{\partial p} / \partial x)_{2}$ critical reflux decreases with increasing $M, \xi / \mu$ and We. The effects of $M, \xi / \mu$, and We on mean velocity and reversal flow are displayed in Figures 4.7, 4.8, and 4.9. The results reveal that the reversal flow increases with increasing $M, \xi / \mu$ and We. 


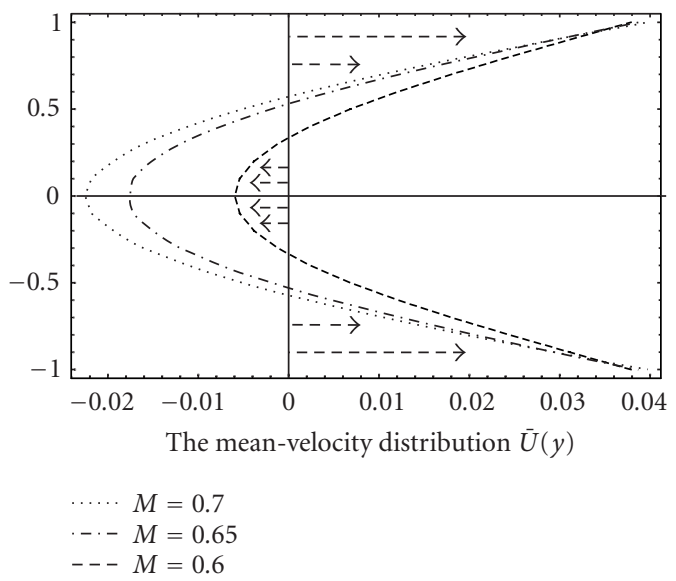

Figure 4.8. Effect of the magnetic parameter $M$ on the mean-velocity distribution and reversal flow for $(\overline{\partial p} / \partial x)_{2}=1, \alpha=0.1, \mathrm{We}=1, \epsilon=0.15, \xi / \mu=1$, and $R=10$.

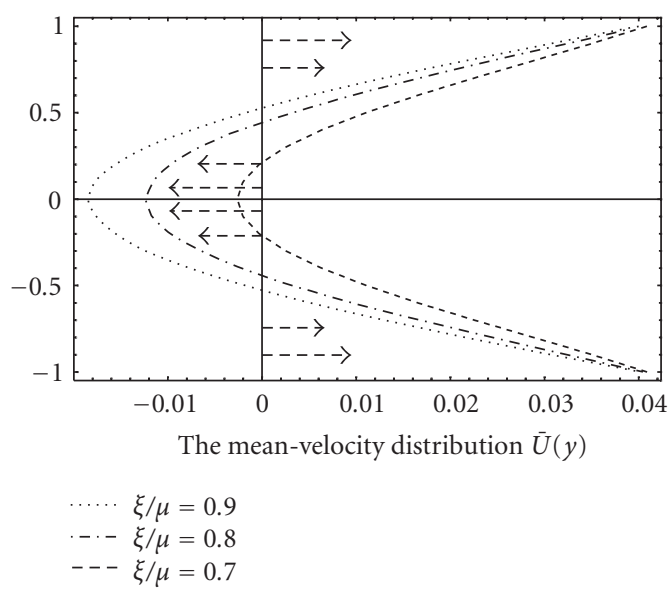

Figure 4.9. Effect of the viscosity ratio $\xi / \mu$ on the mean-velocity distribution and reversal flow for $(\overline{\partial p} / \partial x)_{2}=1, \mathrm{We}=1, M=0.7, \alpha=0.1, \epsilon=0.15$, and $R=10$.

\section{References}

[1] H. L. Agrawal and B. Anwaruddin, Peristaltic flow of blood in a branch, Ranchi Univ. Math. J. 15 (1984), 111-121.

[2] G. Bohme and R. Friedrich, Peristaltic flow of viscoelastic liquids, J. Fluid Mech. 128 (1983), $109-122$.

[3] T. G. Cowling, Magnetohydrodynamics, Interscience Tracts on Physics and Astronomy, no. 4, Interscience, New York, 1957.

[4] A. S. Gupta, On the flow of an electrically conducting fluid near an accelerated plate in the presence of a magnetic field, J. Phys. Soc. Japan 15 (1960), no. 10, 1894-1897. 
[5] T. Hayat, S. Asghar, and A. M. Siddiqui, Stokes' second problem for a Johnson-Segalman fluid, Appl. Math. Comput. 148 (2004), no. 3, 697-706.

[6] T. Hayat, Y. Wang, A. M. Siddiqui, and K. Hutter, Peristaltic motion of a Johnson-Segalman fluid in a planar channel, Math. Probl. Eng. 1 (2003), no. 1, 1-23.

[7] M. Y. Jaffrin and A. H. Shapiro, Peristaltic pumping, Ann. Rev. Fluid Mech. 3 (1971), 13-37.

[8] M. W. Johnson Jr. and D. Segalman, A model for viscoelastic fluid behavior which allows nonaffine deformation, J. Non-Newtonian Fluid Mech. 2 (1977), no. 3, 255-270.

[9] R. W. Kolkka, D. S. Malkus, M. G. Hansen, and G. R. Ierley, Spurt phenomena of the JohnsonSegalman fluid and related models, J. Non-Newtonian Fluid Mech. 29 (1988), 303-335.

[10] D. S. Malkus, J. A. Nohel, and B. J. Plohr, Dynamics of shear flow of a non-Newtonian fluid, J. Comput. Phys. 87 (1990), no. 2, 464-487.

[11] T. C. B. McLeish and R. C. Ball, A molecular approach to the spurt effect in polymer melt flow, J. Polym. Sci. (B) 24 (1986), no. 8, 1735-1745.

[12] Kh. S. Mekheimer, Peristaltic flow of blood under effect of a magnetic field in a non-uniform channels, Appl. Math. Comput. 153 (2004), no. 3, 763-777.

[13] J. C. Misra and S. K. Pandey, A mathematical model for oesophageal swallowing of a food-bolus, Math. Comput. Modelling 33 (2001), no. 8-9, 997-1009.

[14] G. Radhakrishnamacharya, Long wavelength approximation to peristaltic motion of a power law fluid, Rheol. Acta 21 (1982), no. 1, 30-35.

[15] I. J. Rao, Flow of a Johnson-Segalman fluid between rotating co-axial cylinders with and without suction, Internat. J. Non-Linear Mech. 34 (1999), no. 1, 63-70.

[16] I. J. Rao and K. R. Rajagopal, Some simple flows of a Johnson-Segalman fluid, Acta Mech. 132 (1999), no. 1-4, 209-219.

[17] A. H. Shapiro, M. Y. Jaffrin, and S. L. Weinberg, Peristaltic pumping with long wavelengths at low Reynolds number, J. Fluid Mech. 37 (1969), no. 4, 799-825.

[18] A. M. Siddiqui, A. Provost, and W. H. Schwarz, Peristaltic pumping of a second-order fluid in a planar channel, Rheol. Acta 30 (1991), no. 3, 249-262.

[19] A. M. Siddiqui and W. H. Schwarz, Peristaltic flow of a second-order fluid in tubes, J. NonNewtonian Fluid Mech. 53 (1994), 257-284.

[20] D. Srinivasacharya, M. Mishra, and A. R. Rao, Peristaltic pumping of a micropolar fluid in a tube, Acta Mech. 161 (2003), no. 3-4, 165-178.

[21] L. M. Srivastava and R. P. Agrawal, Oscillating flow of a conducting fluid with a suspension of spherical particles, J. Appl. Mech. 47 (1980), 196-199.

[22] L. M. Srivastava and V. P. Srivastava, Peristaltic transport of blood: Casson model_II, J. Biomech. 17 (1984), no. 11, 821-829.

[23] Peristaltic transport of a non-Newtonian fluid: applications to the vas deferens and small intestine, Ann. Biomed. Eng. 13 (1985), no. 2, 137-153.

[24] Peristaltic transport of a power-law fluid: application to the ductus efferentes of the reproductive tract, Rheol. Acta 27 (1988), no. 4, 428-433.

[25] V. K. Sud, G. S. Sekhon, and R. K. Mishra, Pumping action on blood by a magnetic field, Bull. Math. Biol. 39 (1977), no. 3, 385-390.

[26] F. C. P. Yin and Y. C. Fung, Comparison of theory and experiment in peristaltic transport, J. Fluid Mech. 47 (1971), 93-112.

Moustafa Elshahed: Girls College of Education, P.O. Box 3771, Qassim-Unaizah, Saudi Arabia E-mail address: elshahedm@yahoo.com

Mohamed H. Haroun: Faculty of Education, Qassim, Al-Mezneb, Saudi Arabia

E-mail address: hassan6aky@yahoo.com 


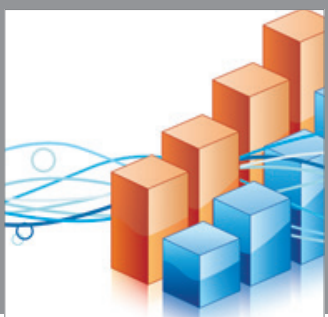

Advances in

Operations Research

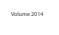

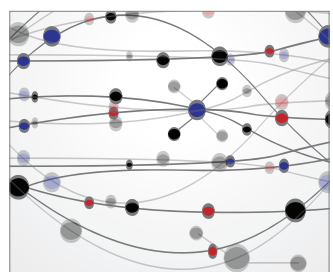

\section{The Scientific} World Journal
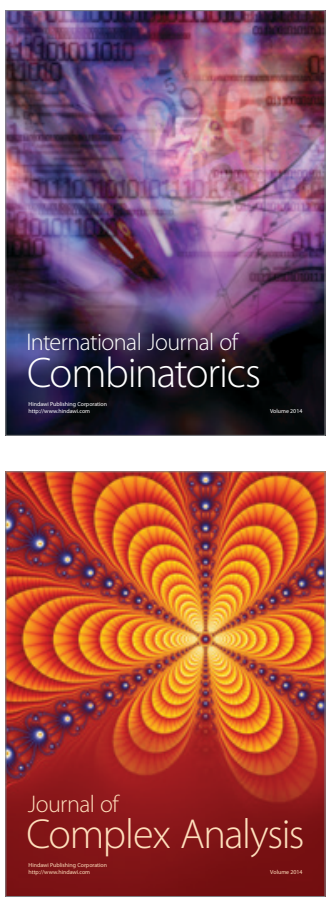

International Journal of

Mathematics and

Mathematical

Sciences
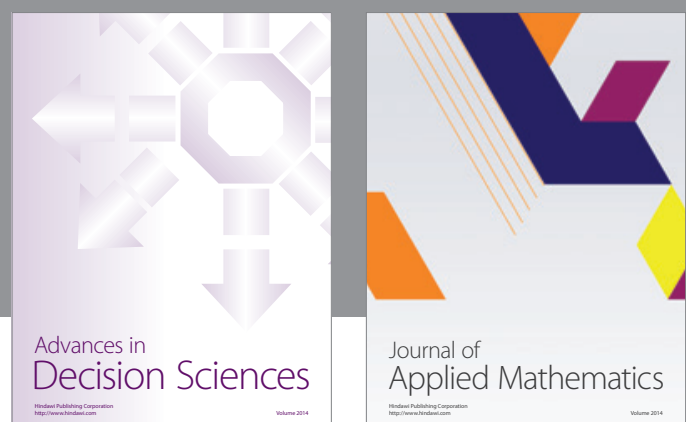

Journal of

Applied Mathematics
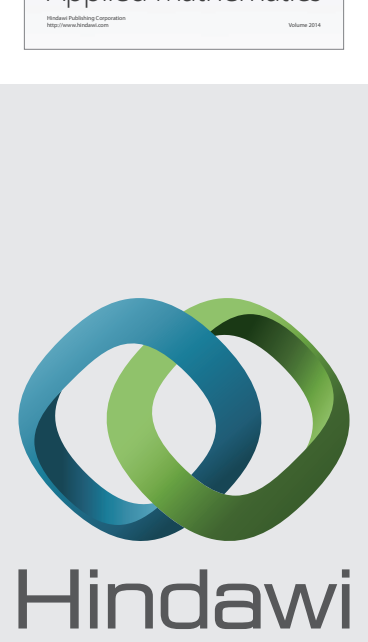

Submit your manuscripts at http://www.hindawi.com
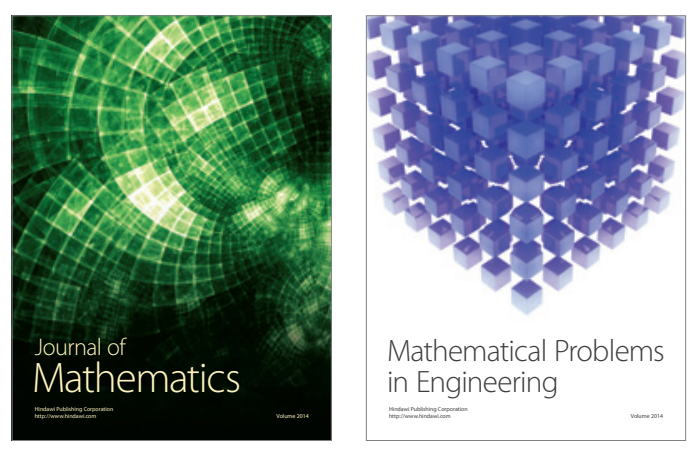

Mathematical Problems in Engineering
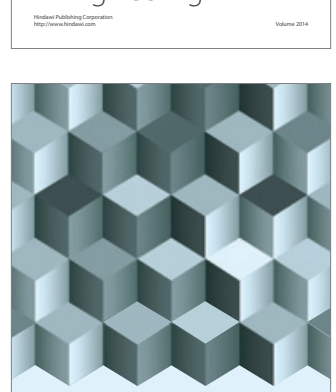

Journal of

Function Spaces
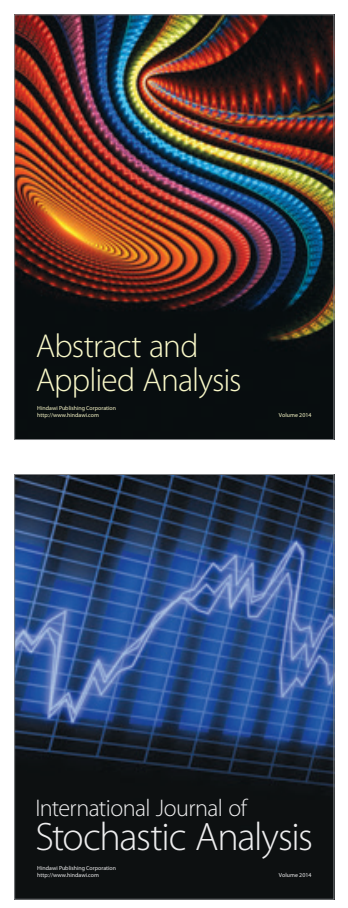

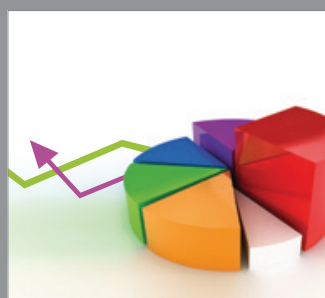

ournal of

Probability and Statistics

Promensencen
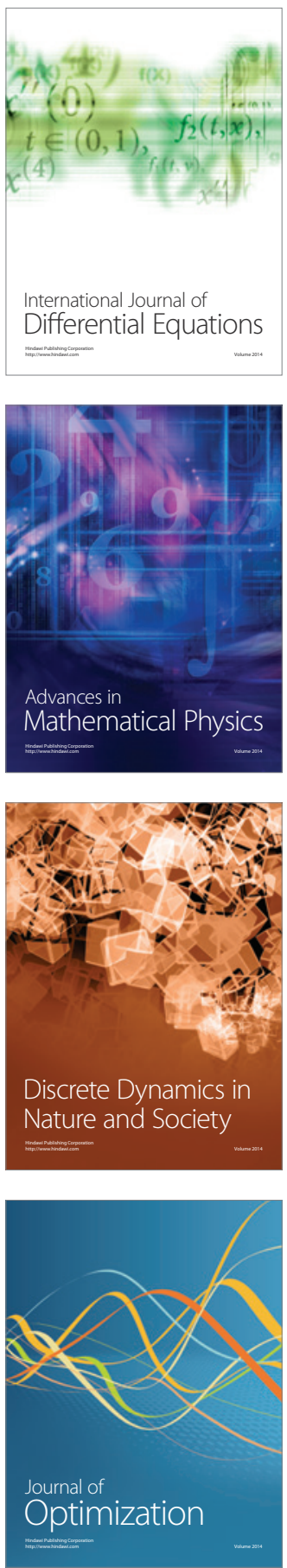